\title{
SUBCILIARY VERSUS SUBTARSAL APPROACH FOR THE MANAGEMENT OF INFRAORBITAL RIM AND ORBITAL FLOOR FRACTURES. AN ANTHROPOMETRIC ANALYSIS
}

\author{
Fatma Ibrahim Mohamed*
}

\begin{abstract}
Purpose: the aim of this study was to assess the anthropometric changes of the morphology of the lower eyelid and the esthetic outcomes after the use of the subciliary (SCA) and subtarsal (STA) approaches in patients with infraorbital rim and orbital floor fractures.

Patients and methods: Twenty-six patients with orbital floor and infraorbital rim fractures were included in the study. They were divided equally into two groups. In group 1 , the fractures of the orbital floor and infraorbital rim were approached via the stepped SCA, whereas in group 2, they were approached via the STA. All the cases were evaluated for the anthropometric changes in the morphology of the lower eyelid and the esthetic outcomes of the scars as well as the postoperative lower eyelid complications (LLCs). A paired sample $t$-test was used to analyze the difference between the operated and non-operated sides, as well as the effect of the surgical approach on the anthropometric measurements.
\end{abstract}

Results: The two approaches provided adequate exposure of the infraorbital rim and orbital floor. STA provided rapid access to the field. The anthropometric analysis revealed that there was no significant difference between the two approaches, except for the Eye fissure index (EFI) and lower iris coverage (LIC) measurements. The SCA had a significant increase in the EFI than the STA. Furthermore, the SCA showed a significant decrease in the measurements of the LIC.

Conclusion: The STA is a minimally invasive incision that provides adequate and direct exposure to the orbital floor and infraorbital rim fractures with more stable periorbital architecture and anthropometric measurements with the minimal incidence of postoperative complications.

KEYWORDS: Subciliary approach (SCA); Subtarsal approach (STA); Anthropometric analysis; Infraorbital rim fractures; Orbital floor fractures; Lower eyelid complications (LLCs).

\footnotetext{
* Associate Professor of Oral and Maxillofacial Surgery, Faculty of Dental Medicine for Girls, Al Azhar University.
} 


\section{INTRODUCTION}

Maxillofacial surgeons are usually dealing with orbital problems such as trauma, pathology, bone reconstruction, and esthetics. ${ }^{(1,2)}$ Orbital fractures represent a high percentage of all facial fractures $(30 \%-40 \%)$, especially those of the orbital floor and orbital rim fractures, which may occur in isolation (blowout fractures) or as a part of associated facial fractures mostly the zygomaticomaxillary complex (ZMC) fracture. ${ }^{(3)}$ For the management of orbital and periorbital fractures, both infraorbital rim and orbital floor can be accessed through transcutaneous and transconjunctival approaches (TCA). The transcutaneous approaches include subciliary (SCA), subtarsal (STA), and infraorbital (IOA) approaches. ${ }^{(2)}$ The selection of the most appropriate surgical approach is guided by the following goals: adequate intraoperative visibility, minimal postoperative scars, and good esthetic outcomes.

Both SCA and STA usually provide sufficient visualization and access to the infraorbital rim and orbital floor fractures; however, they differ in terms of simplicity, the time needed to gain access, and esthetic outcomes. Because of the importance of the eyelids to the facial esthetics, any minor changes in their architecture may have a major impact on patients' esthetics and psychological outcomes. ${ }^{(4)}$ The assessment of such changes is highly desirable, as it may be useful in quantifying the effect of the selected surgical approach on the eyelid morphology. Many anthropometric measurements of the face are available in the literature. ${ }^{(5-10)}$ their benefit in planning, performance, and evaluation of facial surgeries' outcomes are widely recognized. (8-10) However, these anthropometric measurements are not widely used to precisely evaluate the effect of the SCA and STA on the morphology of the lower eyelid. Therefore, the aim of this prospective study was to evaluate the anthropometric changes in the morphology of the lower eyelid, the esthetic outcomes as well as the postoperative lower eyelid complications (LLCs) after the use of the SCA and STA, in patients with orbital and periorbital fractures.

\section{PATIENTS AND METHODS}

\section{Study Design and Population}

This prospective comparative clinical study included 26 patients who suffered from orbital floor and infraorbital rim fractures. They were selected from the outpatient clinic of the Department of Oral and Maxillofacial Surgery, Faculty of Dental Medicine for Girls, Al Azhar University between January 2016 and March 2019. Patients who fulfilled the following inclusion criteria were included in the study: (1) patients who have unilateral orbital floor and rim fractures with unresolved diplopia or indicated for open reduction and internal fixation (ORIF); (2) the surgical approaches were performed by the same surgeon, and (3) lack of maxillofacial deformities of the contralateral eyelid. The patients were excluded if they have the following: (1) undisplaced fractures; (2) extensive soft-tissue damage of the lower eyelid; (3) syndromic or medically compromised patients; (4) history of previous trauma, lesions or surgery in the orbital region or eyelids of the affected or the contralateral side; and (5) those with bilateral orbital and periorbital fractures. The patients were randomly divided into two equal groups. In group 1, the fractures of the orbital floor and infraorbital rim were approached via the stepped SCA, whereas in group 2, they were approached via the STA. In accordance with the Declaration of Helsinki, written informed consent was obtained from all patients, and the local ethics review committee of the Faculty of Dental Medicine for Girls at Al Azhar University approved the study.

\section{Study variables}

The primary predictor variable was the type of surgical approach. The primary outcome variable was the change in the anthropometric measurements of the lower eyelid. The anthropometric 
measurements of the contralateral lower eyelid, in each group, were used as a control to evaluate the changes in the morphology of the operated side. The secondary outcome variables were the esthetic outcome of the scar and the postoperative LLCs which included ectropion, entropion, scleral-show, and canthal malposition. Furthermore, the presence of diplopia, enophthalmos, lagophthalmos (inability to completely close the eyes), limitations in eyeball movements, trichiasis, epiphora, paresthesia, wound infection, plates' exposure, asymmetry of canthal ligaments, keratoconjunctivitis, and subconjunctival injections (the presence of localized congestion along the distribution of scleral-show) were also evaluated. As an additional record, the surgical time that elapsed from the skin incision to the exposure of the fracture was also recorded.

\section{Surgical procedures}

All the surgeries were performed under general anesthesia through nasoendotracheal intubation, at the Department of Oral and Maxillofacial surgery of Al Zahraa hospital, Al Azhar University. To provide adequate hemostasis, $2 \%$ mepivacaine with 1:200,000 epinephrine was injected through the incision line. The two approaches were performed in a standardized manner according to the techniques which were described by Ellis and Zide. ${ }^{(11)}$ A 3-0 silk suture was passed through the margin of the upper and lower eyelids, to be used as a temporary tarsorrhaphy for globe protection and as a Frost suture at the end of the surgery. In group 1, the stepped SCA was performed $2 \mathrm{~mm}$ inferior and parallel to the lower lid lash line. It extended from the medial canthal region, through the skin crease, to the lateral aspect of the orbit. The skin and orbicularis oculi muscle dissection were performed in a stepped manner. The dissection was continued to the level of the infraorbital rim. Once the rim was reached, an incision through the periosteum was performed to gain access to the orbital floor, and then the periosteum was reflected with a freer elevator.
In group 2, the STA was made in the subtarsal fold, and it was extended laterally into one of the resting skin tension lines along the lateral aspect of the orbit. The orbicularis oculi muscle was bluntly dissected in the same plane with blunt-tipped scissors to the level of the orbital rim. The periosteum just below the infraorbital rim was incised and reflected to expose the orbital floor and infraorbital rim. In both groups, malleable retractors with different sizes were used to retract the eye globe and expose the orbital floor. Both approaches were performed without any medial extension to avoid disruption of the nerve supply of the orbicularis oculi muscle. In cases of ZMC fractures, the frontozygomatic suture was reached through the lateral eyebrow approach. In all patients, after achieving an accurate reduction of the fractures, $2.0 \mathrm{~mm}$ miniplates and screws were used to provide adequate fixation, depending on the requirements of each case. Wound closure was performed in two layers; the periosteum was closed with a 5-0 resorbable suture and the skin incisions were closed with 6-0 nylon sutures. The Frost suture was removed 4 - 7 days postoperatively.

\section{Data collection}

The following data were recorded in the patients' medical charts: patients' age, sex, photographs, etiology and site of fracture, associated maxillofacial injuries, preoperative ophthalmic symptoms (facial paresthesia, diplopia, and enophthalmos), radiographic findings, the time interval between trauma occurrence and treatment, surgical details, and postoperative complications. All the patients were subjected to pre- and postoperative CT scans and ophthalmic examination by the same ophthalmologist to evaluate all clinical and radiographic signs and symptoms before and after surgeries.

To evaluate the morphology of the eyelids; the anthropometric measurements of the lower eyelid, for both operated and non-operated sides $(9,10)$ and the esthetic appearance of the scar were postoperatively recorded six months after surgery. 
Both variables were recorded by two examiners who were blind with the research and they were not informed about the operated side. The esthetic appearance of the scar was evaluated according to the scale of Feldman et al., ${ }^{(12)}$ which ranges from score 0 to 4 (Score 0 , not visible; score 1, barely visible; score 2 , noticeable; score 3 , very noticeable; score 4 , extremely noticeable). The LLCs were also recorded for all patients at the end of the first week, at the first, third, and sixth months postoperatively.

\section{Anthropometric measurements}

The anthropometric analysis was performed according to many authors. ${ }^{(5-10,13,14)}$ To record these variables, standard photographs were taken by a professional photographer. The patients were asked to set while their facial midline was perpendicular to the ground and asked to keep their eyes open and look straight to the lens of the camera. All the photographs were standardized to the size of $5 \times 10$ inch. The photographic analysis was performed by using the measurement tool of Adobe Photoshop (Adobe, San Jose, CA, USA). All the measurements were standardized against the measured width of the iris which is $12 \mathrm{~mm}$ on average in humans. On each photograph (Figure 1), the anthropometric landmarks, distances, and angles which are summarized in Tables 1,2, 3 were identified and drawn.

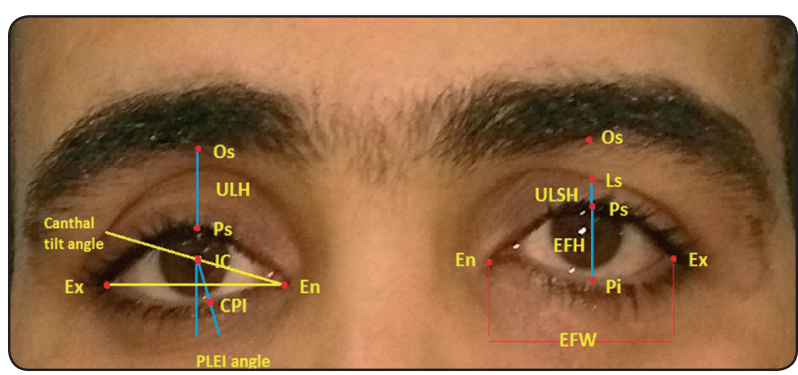

Fig. (1) Anthropometric landmarks, distances, and angles used in this study

TABLE (1) Definition of the Anthropometric landmarks

\begin{tabular}{|c|c|}
\hline $\begin{array}{l}\text { Palpebrale superioris } \\
\text { (Ps) }\end{array}$ & $\begin{array}{l}\text { The point in the mid-portion of the free margin of the upper eyelid constructed by extending a line from } \\
\text { the mid-pupil to the upper eyelid }\end{array}$ \\
\hline $\begin{array}{l}\text { Palpebrale inferioris } \\
\text { (Pi ) }\end{array}$ & $\begin{array}{l}\text { The point in the mid-portion of the free margin of the lower eyelid constructed by dropping a line from } \\
\text { the mid-pupil to the lower eyelid }\end{array}$ \\
\hline Endocanthion (En) & The point at the inner commissure of the eye fissure (the inner junction of the upper and lower eyelids) \\
\hline Exocanthion $(\mathbf{E x})$ & The point at the outer commissure of the eye fissure (the outer junction of the upper and lower eyelids) \\
\hline Ic & Iris Centre \\
\hline LS & Eyelid sulcus \\
\hline Os & Orbitale superioris (highest point on the lower border of the eyebrow) \\
\hline $\mathbf{C P i}$ & Corneal palpebral inferior contact point (contact of the lower eyelid and cornea) \\
\hline
\end{tabular}

TABLE (2) The anthropometric distances used in this study

\begin{tabular}{|ll|}
\hline Eye fissure height (EFH) & The distance between the Ps and Pi \\
Eye fissure width (EFW) & The distance between the En and Ex \\
Upper eyelid sulcus height (ULSH) & The distance between the LS and Ps \\
Upper eyelid height (ULH) & The distance between the Os and Ps \\
Iris Diameter (ID) & The horizontal distance between the white margin of the iris on one side to that on the \\
& other side \\
Upper iris radius visible (UIRv) & The vertical distance between the center of the pupil and the upper visible part of the iris \\
Lower iris radius visible (LIRv) & The vertical distance between the center of the pupil and the lower visible part of the iris \\
\hline
\end{tabular}


TABLE (3) The anthropometric analysis

\begin{tabular}{|c|c|}
\hline Data & Description \\
\hline $\begin{array}{l}\text { Eye Fissure In- } \\
\text { dex }(\text { EFI, \%) }\end{array}$ & $\begin{array}{l}\text { The percentage of the ratio between the } \\
\text { EFH and EFW }\end{array}$ \\
\hline $\begin{array}{l}\text { Upper eyelid } \\
\text { sulcus height } \\
\text { ULSH }(\%)\end{array}$ & $\begin{array}{l}\text { The percentage of the distance between } \\
\text { the Ls and Ps to the distance between } \\
\text { OS-Ps }\end{array}$ \\
\hline $\begin{array}{l}\text { Upper iris cov- } \\
\text { erage (UIC, \%) }\end{array}$ & $\begin{array}{l}\text { - The percentage of that part of the iris } \\
\text { which is covered by the upper eyelid as } \\
\text { a percentage of the total ID } \\
\text { - It was measured by halving the ID, } \\
\text { and then subtracting the UIRv }\end{array}$ \\
\hline $\begin{array}{l}\text { Lower iris cov- } \\
\text { erage (LIC, \%) }\end{array}$ & $\begin{array}{l}\text { - The percentage was calculated by the } \\
\text { same method of the UIC but with the } \\
\text { reference to the lower eyelid and the } \\
\text { lower part of the iris } \\
\text { - The negative results are obtained if } \\
\text { there was a scleral show or ectropion }\end{array}$ \\
\hline $\begin{array}{l}\text { Position of the } \\
\text { lower eyelid to } \\
\text { the lower iris } \\
\left(\text { PLEI }^{\circ}\right)\end{array}$ & $\begin{array}{l}\text { - It is the angle between the lower } \\
\text { eyelid and Ic } \\
\text { - This angle is formed between the } \\
\text { vertical line which passes through } \\
\text { Ic and that line which connects the } \\
\text { Ic with the contact point between the } \\
\text { lower eyelid and cornea (CPI) } \\
\text { - Negative values: If the angle was } \\
\text { medially deviated } \\
\text { - Positive values: If the angle was } \\
\text { laterally deviated }\end{array}$ \\
\hline Canthal tilt $\left(^{\circ}\right)$ & $\begin{array}{l}\text { It is the intercanthal fissure inclination } \\
\text { which is the angle between the EFW } \\
\text { and the horizontal line which passes } \\
\text { through the En and Ic }\end{array}$ \\
\hline
\end{tabular}

\section{Statistical analysis}

The collected data were recorded, tabulated, and analyzed statistically. The data were analyzed with Microsoft Office XP (Excel) and SPSS version 15.00 software (SPSS Inc., Chicago, IL, USA). Parametric data were expressed as the mean \pm standard deviation (SD), and non-parametric data were expressed as the number and percentage of the total. A paired sample $t$-test was used to analyze the difference between the operated and nonoperated sides and compare the effect of the surgical approach on the anthropometric measurements and the scar esthetic score in the two groups. $P$ values $\leq 0.05$ were considered statistically significant. To correlate between the effect of the demographic data of the patients and the surgical approach on the LLCs, chi²-test was conducted.

\section{RESULTS}

The patients' population consisted of 16 males $(61.5 \%)$ and 10 females $(38.5 \%)$ with a male to female ratio 1.6: 1 . The patients' age ranged from 17 to 56 years (mean $32 \pm 11.7$ years) at the time of surgery. Twenty patients underwent the corrective surgery within one week of the trauma and the rest of the patients underwent the surgery within one month after the trauma. The most frequent cause of the fracture was interpersonal violence (IPV) in 14 cases (54\%), followed by the road traffic accident (RTA) in six patients $(23 \%)$, falls were reported in three cases $(11.5 \%)$, and sports injuries were also recorded in three cases $(11.5 \%)$. Out of 26 fractures, only 9 patients had isolated orbital floor fractures, whereas 17 cases were associated with concomitant maxillofacial fractures mostly the ZMC fractures. The descriptive characteristics of both groups are presented in Table 4. All the preoperative ophthalmic examinations showed that there was no history of lid injuries. Moreover, there was no disturbance in the visual acuity, pupil size, and reactivity, either pre- or postoperatively.

\section{Intraoperative Results}

The two incisions provided adequate exposure of the infraorbital rim and orbital floor. The mean operating time from the incision to the exposure of the fracture was $13.7 \pm 2.8$ minutes for SCA and $10.1 \pm 1.7$ minutes for STA (Table 5) $(P$-value $=0.001)$. The reconstruction of the orbital floor was carried out with titanium mesh in 11 patients $(42.3 \%)$, and the preformed titanium plates were used in 10 patients $(38.5 \%)$. Exploration was only performed in five patients $(19.2 \%)$. 
TABLE (4) Demographic features of the patients

\begin{tabular}{|c|c|c|c|}
\hline Study variables* & $\begin{array}{l}\text { Group 1 } \\
\text { (SCA) }\end{array}$ & $\begin{array}{c}\text { Group } 2 \\
\text { (STA) }\end{array}$ & $P$-value \\
\hline Gender & & & 0.4 \\
\hline Female & 6 & 4 & \\
\hline Male & 7 & 9 & \\
\hline Age & $28.3 \pm 10.6$ & $36 \pm 11.3$ & 0.09 \\
\hline \multicolumn{4}{|l|}{ Etiology } \\
\hline IPV & $7(53.8)$ & $7(53.8)$ & 1 \\
\hline RTA & $4(30.8)$ & $2(15.4)$ & 0.4 \\
\hline Falls & $1(7.7)$ & $2(15.4)$ & 0.6 \\
\hline Sports & $1(7.7)$ & $2(15.4)$ & 0.6 \\
\hline \multicolumn{4}{|l|}{ Type of fracture } \\
\hline $\mathrm{ZMC}$ & $5(38.5)$ & $6(46.1)$ & 0.7 \\
\hline $\begin{array}{l}\text { Isolated orbital } \\
\text { floor and rim }\end{array}$ & $6(46.1)$ & $3(23.1)$ & 0.2 \\
\hline Le Fort II & $2(15.4)$ & $4(30.8)$ & 0.4 \\
\hline
\end{tabular}

Data presented as mean $\pm S D$ or numbers, with percentages in parentheses.

All p-values were non-significant $(p>0.05)$

TABLE (5) Intraoperative surgical details

\begin{tabular}{|c|c|c|c|}
\hline & $\begin{array}{l}\text { Group } 1 \\
\text { (SCA) }\end{array}$ & $\begin{array}{c}\text { Group } 2 \\
\text { (STA) }\end{array}$ & $P$-value \\
\hline $\begin{array}{l}\text { Incision time } \\
(\text { mean } \pm \text { SD, minutes })\end{array}$ & $13.7 \pm 2.8$ & $10.1 \pm 1.7$ & $0.001 *$ \\
\hline $\begin{array}{l}\text { Orbital floor repair } \\
\text { (patients) }\end{array}$ & & & \\
\hline Titanium mesh & $7(53.8)$ & $4(30.8)$ & 0.2 \\
\hline Preformed Titanium plate & $4(30.8)$ & $6(46.1)$ & 0.4 \\
\hline Exploration & $2(15.4)$ & $3(20)$ & 0.6 \\
\hline
\end{tabular}

Data presented as mean $\pm S D$ or numbers, with percentages in parentheses.

* Significant p-value $\leq 0.05$

\section{Postoperative Results}

The postoperative CT scans revealed satisfactory reduction and fixation of the fractures. In addition, there were no defects of the orbital floor, no herniation of the ocular contents into the maxillary sinus, and no evidence of zygomatic asymmetry in the cases of ZMC fractures. None of the patients had infection, hardware exposure, persistent diplopia, or enophthalmos. In all the patients, the postsurgical lid edema seemed to resolve within the first month of surgery.

\section{Anthropometric Analysis}

The anthropometric measurements of the operated side were changed as compared to the normal side; however, the results did not show any statistical significance in each group $(P$-value $>$ 0.05 ) as shown in Table 6. This revealed that the two incisions provide proper postoperative esthetic outcomes of the operated lower eyelid when compared to the normal side. When comparing the anthropometric measurements of the operated lower eyelid in both groups, the results revealed that the difference between these two incisions was not significant, except for the EFI and LIC measurements. The mean of the EFI measurements increased in all operated sides. The SCA showed a significant increase in the EFI variable than the STA, $(P$-value $=0.045)$. Furthermore, the SCA showed a significant decrease in the measurements of the LIC. This change was also greater with the SCA than that with the STA $(P$-value $=0.04)$.

\section{Results of LLCs}

The postoperative ectropion was observed in three patients $(23.1 \%)$ with the SCA, while it was reported in one patient $(7.7 \%)$ in the STA group. No significant difference was observed between the two groups regarding this complication (Table 6). The postoperative ectropion resolved by time without surgical intervention. Four patients $(30.8 \%)$ in the SCA group suffered from scleral-show; three of them complained of subconjunctival injections. One case in group 2 also suffered from scleral-show. Epiphora was also observed in three patients; two patients in the SCA group and one subject in the STA group, with no statistically significant difference. 
TABLE (6) The anthropometric measurements of the operated and un-operated sides and LLCs of the two groups

\begin{tabular}{|c|c|c|c|c|c|c|c|}
\hline & \multicolumn{3}{|c|}{ Group 1 (SCA) } & \multicolumn{3}{|c|}{ Group 2 (STA) } & \multirow{2}{*}{$\begin{array}{c}P \text {-value of } \\
\text { both groups }\end{array}$} \\
\hline & Affected & $\begin{array}{c}\text { Non- } \\
\text { affected }\end{array}$ & $p$-value & Affected & Non-affected & $p$-value & \\
\hline EFI (\%) & $34.2 \pm 4.1$ & $32 \pm 4$ & 0.2 & $31 \pm 3.4$ & $30.8 \pm 2.2$ & 0.9 & $0.045^{*}$ \\
\hline $\begin{array}{l}\text { ULSH } \\
(\%)\end{array}$ & $34.3 \pm 5.4$ & $31.7 \pm 4.8$ & 0.2 & $31.8 \pm 3$ & $30.3 \pm 2.4$ & 0.2 & 0.2 \\
\hline UIC (\%) & $17.8 \pm 2.4$ & $17.5 \pm 2.1$ & 0.7 & $18.8 \pm 4.4$ & $17.4 \pm 2.5$ & 0.4 & 0.5 \\
\hline LIC (\%) & $0.8 \pm 2.1$ & $2 \pm 1.8$ & 0.1 & $2.4 \pm 1.3$ & $2.7 \pm 1.3$ & 0.5 & $0.04 *$ \\
\hline PLEI $\left({ }^{\circ}\right)$ & $-2.2 \pm 5.1$ & $-0.5 \pm 3.5$ & 0.07 & $-0.6 \pm 2.7$ & $-0.45 \pm 3.4$ & 0.8 & 0.06 \\
\hline Canthal tilt $\left({ }^{\circ}\right)$ & $2 \pm 3.2$ & $1.6 \pm 3$ & 0.4 & $1.4 \pm 3.4$ & $1.6 \pm 4.8$ & 0.8 & 0.3 \\
\hline Scleral- show (\%) & 30.8 & 15.4 & 0.4 & 7.7 & 7.7 & 1 & 0.1 \\
\hline Ectropion (\%) & 23.1 & 0 & - & 7.7 & 0 & - & 0.3 \\
\hline Entropion & 0 & 0 & - & 0 & 0 & - & - \\
\hline Epiphora (\%) & 15.4 & 0 & - & 7.7 & 0 & - & 0.6 \\
\hline
\end{tabular}

Data presented as mean $\pm S D$ or percentages.

* Significant p-value $\leq 0.05$

The condition was transitory and gradually resolved during the follow-up period. The $\mathrm{chi}^{2}$-test revealed that there was no significant correlation between the patient's age, sex, the time interval between the trauma and surgical interventions, operative time, and the type of surgical approach with the incidence of LLCs among the two groups. Regarding the affection of the infraorbital nerve, 12 patients (46\%) suffered from preoperative impairment of the nerve function. Seven out of 12 patients included in group 1 and five cases included in group 2. The sensory disturbance was transient and completely resolved within three months after surgeries.

\section{Scar Esthetic Score}

A closer look at the incision site revealed that the mean scar score was $0.61 \pm 0.9$ for group 1 and $1 \pm 1.2$ for group 2 (Table 7). This reveals a nonsignificant difference between the SCA and STA $(P$-value $=0.4)$. None of the patients developed scar hypertrophy, except one case who was included in the STA group (score=4). Nevertheless, all the patients were satisfied with the postoperative outcomes, both esthetically and functionally. A photographic description of two patients of each group was shown in Fig. 2.

TABLE (7) The results of the scar esthetic score

\begin{tabular}{|c|c|c|c|}
\hline & $\begin{array}{c}\text { Group } 1 \\
\text { (SCA) }\end{array}$ & $\begin{array}{c}\text { Group } 2 \\
\text { (STA) }\end{array}$ & $P$-value \\
\hline Score 0 & $8(66.7 \%)$ & $6(46.7 \%)$ & \\
\hline Score 1 & $3(20 \%)$ & $4(26.7 \%)$ & \\
\hline Score 2 & $1(6.7 \%)$ & $1(6.7 \%)$ & \\
\hline Score 3 & $1(6.7 \%)$ & $1(6.7 \%)$ & \\
\hline Score 4 & $0(0)$ & $1(13.3 \%)$ & \\
\hline Mean \pm SD & $0.61 \pm 0.9$ & $1 \pm 1.2$ & 0.4 \\
\hline
\end{tabular}

Data presented as mean $\pm S D$ or numbers, with percentages in parentheses.

$P$-value was non-significant $(p>0.05)$ 


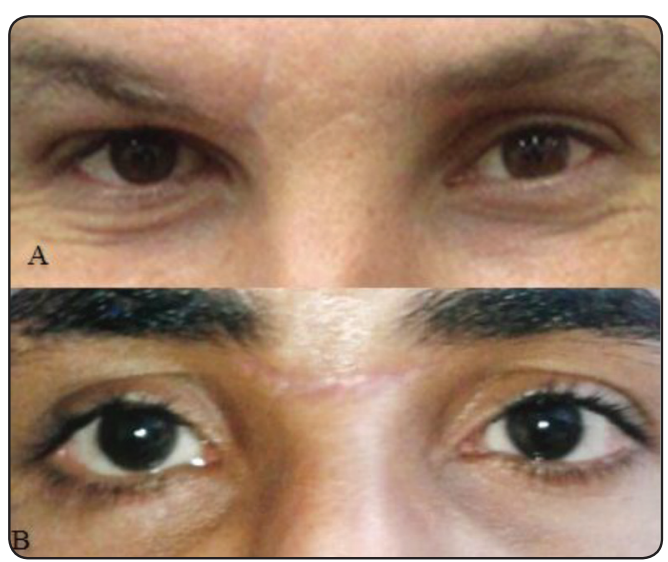

Fig. (2) Standardized photographs of patients treated through A. Subciliary approach (right side), B. Subtarsal approach (right side)

\section{DISCUSSION}

Orbital and periorbital injuries frequently require surgical treatment. Indications for orbital floor repair include muscle entrapment, enophthalmos, and displaced fractures as evidenced on a CT scan. ${ }^{(3)}$ IPV is the most frequent cause of orbital fractures in this study, which is in line with the results of many studies. (15,16) On contrary, MVA was considered the main etiology of injury in facial trauma, in other studies. ${ }^{(3,17)}$ The frequency of fracture was observed in males more than females (male: female ratio 1.6:1). This ratio compares favorably with many authors. ${ }^{(3,16-18)}$ Out of the 26 fractures, only 9 patients had isolated orbital floor fractures while nearly two-thirds of the cases were associated with concomitant maxillofacial injuries, mainly the ZMC fractures. The same results were also reported by Schmäl et al. ${ }^{(3)}$

When approaching the face surgically, the cosmetic outcome is an important factor that should be taken into consideration. As the eye and lid area are important determinants of facial esthetics, the surgical access to this region has its unique challenges. Usually, improper placement of orbital incisions will result in both functional and esthetic consequences. Many factors should be considered when selecting a surgical approach to the infraorbital rim and orbital floor. The patient esthetic expectations, the rates of complications that accompany the selected approach, the surgeons' experience, the site and extension of the ortbital fracture are considered important factors that give the reasons why one surgical approach might be selected over the other.

The results of this study revealed that the two incisions provide adequate surgical exposure to the infraorbital rim and orbital floor fractures without encroachment on the lateral canthal ligament and the lacrimal sac, as also reported by many authors. (19,20,21) Moreover, the STA provides more rapid access to the surgical field as it only requires a mean of $10.1 \pm 1.7$ minutes. This could be due to the easily identified lower eyelid anatomy during dissection with adequate surgical exposure which facilitated the placement of the plates and closure of the incision. Whereas the mean of the SCA operating time was $13.7 \pm 2.8$ minutes, which is significantly longer than the STA.

Several anthropometric measurements for the periorbital region have been described in the literature. ${ }^{(5,6)}$ The anthropometric analysis was used to identify the morphological outcomes of the two approaches. All the anthropometric measurements are affected by the type of incision, but their effect did not show significant differences between the operated and contralateral sides, as observed by many authors. ${ }^{(9,10)}$ Furthermore, there are no significant differences between the two incisions regarding the anthropometric measurements of the operated lower eyelid, except for the EFI $(P$-value $=$ $0.045)$ and LIC $(P$-value $=0.04)$. Regarding these two measurements, the SCA showed significant alteration in the shape of the lower eyelid when compared to the STA, which is reflected by the increase in the value of the EFI and the decrease in the value of the LIC. This alteration in the lower eyelid morphology is due to lower eyelid retraction. The same results were also observed by Raschke et al., ${ }^{(9,10)}$ who reported that the EFI was increased and the LIC was decreased following the use of SCA. Therefore, the results of this study revealed that the 
STA should be used whenever possible because it is considered cosmetically acceptable when concealed within a resting skin tension line. Furthermore, it is considered less risky in matters of the eyelid retraction than SCA. Many studies support these findings. ${ }^{(22,23)}$

The changes in the EFI and LIC are mainly due to the change in the position of the Pi and Ex points which are manifested as the inferior retraction of the lower eyelid and the medial displacement of the Ex point. According to Appling et al., ${ }^{(23)}$ Ex point is medially displaced in the cases of lateral canthotomy which is usually performed with the TCA. However, in this study, the Ex point was displaced medially in a significant number of patients in the two groups. This suggested that the change in the position of both points (Pi and Ex) is under the effect of the scar contraction. The same observation was also discussed by Nunu et al. ${ }^{(24)}$

To adequately describe the shape of the eyelids, two angles were evaluated (canthal tilt and PLEI). Both angles exhibit a significant impact on the periorbital appearance as reported by many authors. (7,14) Normally, the lateral canthus lays $10-15^{\circ}$ (25) or $2 \mathrm{~mm}{ }^{(26)}$ above the medial canthus, otherwise, a sad look may be the result of a negative canthal tilt. ${ }^{(14)}$ The PLEI describes the normal contact point of the lower palpebra to the limbus corneae at the 6 o'clock position. ${ }^{(14)}$ The results of this study showed that the two angles were not influenced by the two approaches.

Regarding the results of the esthetic appearance of the scars, the risk of perceiving a noticeable scar was higher for the STA in comparison to SCA with a non-significant statistical difference. This is in accordance with the results of many authors. (12,27) The reason for the increased risk of the noticeable scars with STA is owed to the vertical level of the skin incision. It was reported that the esthetic appearance of the scars seemed to be improved when it is closer to the ciliary margin. ${ }^{(2,19)}$ This is because the skin of the eyelid is thinner and more delicate as it becomes closer to the eyelashes, so it is not predisposed to edema and development of visible scars. ${ }^{(19)}$ De Riu et al., ${ }^{(28)}$ and Salgarelli et al., ${ }^{(22)}$ reported that the SCA also has a high risk of noticeable scars which is in contrast to the results of this study and the results of Feldman et al. (12) This controversy between studies could be due to the method and time of the evaluation during the follow-up period, the age of the patients, and the accuracy of the wound closure as also reported by Kelahmetoglu et al. ${ }^{(29)}$ The results of this study demonstrated that all the scars that were encountered with the STA were acceptable for the patients, and no patient requested a scar revision. So, STA is considered to offer a favorable esthetic outcome.

Regarding the rate of LLCs, many studies compared the two incisions outcomes and reported that although the SCA is more a straightforward technique, it carries the highest rate of the LLCs than the STA. ${ }^{(27,30,31)}$ The scleral show and ectropion are considered the most common form of the eyelid distortion following the SCA, representing 18.8\% and $14 \%$, respectively. ${ }^{(21,27)}$ In contrast, the rate of scleral-show and ectropion for the STA has been reported to be much less, where the incidence of these complications ranged from 4.4 to $8 \%$ and 0 to $3.8 \%$, respectively. ${ }^{(17,27)}$

In this study, comparable results were also observed regarding the scleral-show and ectropion. The highest complications rate was noticed in group 1 (SCA) where three patients $(23.1 \%)$ suffered from ectropion and four patients $(30.8 \%)$ had scleral show. Nevertheless, the anthropometric measurements of the contralateral lower eyelid revealed that two out of those four patients also had scleral-show. Many authors reported similar results. ${ }^{(21,27,30,31)}$ On contrary, other studies showed a relatively lower rate of ectropion with the SCA than the results of this study. ${ }^{(9,10,28,32)}$ In group 2 (STA), only one patient $(7.7 \%)$ had scleral-show in both affected and normal sides. Moreover, the same patient also suffered from ectropion; this is in accordance with the findings of Rohrich et al. ${ }^{(33)}$ who observed that 
the STA is accompanied by a lower incidence of ectropion when it is compared to the SCA.

According to Raschke et al, ${ }^{(9,10)}$ it is important to record the rate of scleral-show of the contralateral side because it may provide information on the preoperative morphology of the operated side, as followed in this study. Therefore, the results of this study revealed that scleral-show only present in two cases of group 1 and the other three cases (two patients in group 1 and one case in groups 2) had normal scleral-show of both sides. Both scleral-show and ectropion, in this study, were considered minor and did not represent a clinical problem for any of the patients and resolved by time as also reported by Liapakis et al. ${ }^{(34)}$ This is owed to the use of the postoperative Frost suture which helps in stretching the orbital septum, retrap the skin of the eyelid, and counteract the scar retraction by lengthening the lower eyelid when it is taped to the forehead. ${ }^{(35)}$

The highest incidence of the postoperative scleral-show, ectropion, and the significant changes in the anthropometric measurements (EFI and LIC) with the SCA, indicate its negative effect on the skin of the lower eyelid. Both SCA and STA are cutaneous incisions, but the path and length of their dissection through the lower eyelid anatomical layers completely differ. The skin incision of the SCA is made just below the eyelashes, so the subcutaneous dissection leads to a very thin skin flap which is a predisposing factor for the contraction of the scar, scleral-show, and ectropion. Whereas that of the STA is placed about 4 to $6 \mathrm{~mm}$ below the lower eyelid margin and runs diagonally in a laterocaudal direction. This provides a thicker skin flap that can resist the occurrence of scleral-show and ectropion. Many other factors may be contributed to the occurrence of scleral-show and ectropion including edema, hematoma, loss of muscle tonus and adhesions between the orbital septum, orbicularis oculi muscle, and surrounding tissue..$^{(9,10,21,32)}$ Furthermore, Subramanian et al., ${ }^{(19)}$ found in their study an association between postoperative ectropion and the tendency towards senile ectropion. The same find- ing was also observed in this study because most of the patients who suffered from postoperative ectropion were older than 45 years.

The limitations of this study are the relatively small sample size and the short-term follow up of the surgical outcomes. Also, different types of osteosynthesis were used in this study, and this may affect the eventual outcome. Nevertheless, this study has many points of strength including; it was a single-institution experience, evaluated multiple outcomes, and it has a comparative nature.

\section{CONCLUSION}

The STA is considered a minimally invasive incision that provides adequate and direct access to the infraorbital rim and orbital floor fractures with more stable periorbital architecture and anthropometric measurements with lower incidence of postoperative complications when it is compared to the SCA. Therefore, STA is recommended as a favorable and routine approach to the infraorbital rim and orbital floor fractures.

\section{Funding sources:}

This research did not receive any specific grant from funding agencies in the public, commercial, or not-for-profit sectors.

\section{Funding disclosures:}

There are no funding disclosures from any authors.

\section{Conflict of interest:}

No conflicts of interest.

The author would like to acknowledge Dr. Ghada Amin Khalifa, Professor of Oral and Maxillofacial Surgery, Faculty of Dental Medicine for Girls, Al Azhar University, for her tremendous educational support. Special thanks to Dr. Susan Abdel-Hakim Hassan, Professor of Oral and Maxillofacial Surgery, Faculty of Dental Medicine for Girls, AlAzhar University for her assistance and performance during the surgeries. 


\section{REFERENCES}

1. Kushner GM: Surgical approaches to the infraorbital rim and orbital floor: The case for the transconjunctival approach. J Oral Maxillofac Surg 64: 108-110, 2006

2. Wilson S, Ellis E: Surgical approaches to the infraorbital rim and orbital floor: The case for the subtarsal approach. J Oral Maxillofac Surg 64:104-107, 2006

3. Schmäl F, Basel T, Grenzebach UHThiede O, Stoll W: Preseptal transconjunctival approach for orbital fracture repair: Ophthalmologic results in 209 patients. Acta OtoLaryngologica 126: 381-389, 2006

4. Bogusiak K, Arkuszewski P: Characteristics and epidemiology of zygomaticomaxillary complex fractures. J Craniofac Surg 21: 1018-1023, 2010

5. Farkas LG, Katic MJ, Forrest CR, Alt KW, Bagic I, Baltadijev G: International anthropometric study of facial morphology in various ethnic groups/races. J Craniofac Surg 16: 615-646, 2005.

6. Farkas LG, Katic MJ, Forrest LG: Comparison of craniofacial measurements of young adult African-American and North American white males and females. Ann Plast Surg 59: 692-698, 2007.

7. Codner MA, Wolfli JN, Anzarut A: Primary transcutaneous lower blepharoplasty with routine lateral canthal support: a comprehensive 10-year review. Plast Reconstr Surg 121: 241-50, 2008.

8. Raschke GF, Bader RD, Rieger UM, Schultze-Mosgau S: Photo-assisted analysis of blepharoplasty results. Ann Plast Surg 66: 328-333, 2011.

9. Raschke GF, Rieger UM, Bader RD, Guentsch A, Schaefer O, Schultze-Mosgau S. The zygomaticomaxillary complex fracture: an anthropometric appraisal of surgical outcomes. J Craniomaxillofac Surg; 41: 331-337, 2013.

10. Raschke G, Djedovic G, Peisker A, Wohlrath R, Rieger U, Guentsch A, Gomez-Dammeier M, Schultze-Mosgau S: The isolated orbital floor fracture from a transconjunctival or subciliary perspective-A standardized anthropometric evaluation. Med Oral Patol Oral Cir Bucal 21: e111-117, 2016.

11. Ellis E, Zide MF: Surgical approaches to the facial skeleton. Philadelphia: Lippincott \& Wilkins, 2006

12. Feldman EM, Bruner TW, Sharabi SE, Koshy JC, Hollier LH Jr: The Subtarsal Incision: Where Should It Be Placed? J Oral Maxillofac Surg 69: 2419-2423, 2011
13. Starck WJ, Griffin JE Jr, Epker BN: Objective evaluation of the eyelids and eyebrows after blepharoplasty. J Oral Maxillofac Surg 54: 297-302, 1996.

14. Biesman BS: Blepharoplasty. Semin Cutan Med Surg 18:129-138, 1999.

15. Higashino $\mathrm{T}$, Hirabayashi $\mathrm{S}$, Eguchi $\mathrm{T}$, Kato $\mathrm{Y}$ : Straightforward factors for predicting the prognosis of blow-out fractures. J Craniofac Surg 22: 1210-1214, 2011

16. Kasaee A, Mirmohammadsadeghi A, Kazemnezhad F, Eshraghi B, Akbari MR: The predictive factors of diplopia and extraocular movement limitations in isolated pure blow-out fracture. J Curr Ophthalmol 29: 54-58, 2017

17. Baqain, Malkawi Z, Hadidi A, Rajab LD: Subtarsal Approach for Orbital Floor Repair: A Long-Term FollowUp of 12 Cases in a Jordanian Teaching Hospital. J Oral Maxillofac Surg 66: 45-50, 2008

18. Ramphul A, Hoffman G: Does preoperative diplopia determine the incidence of postoperative diplopia after repair of orbital floor fracture? An institutional review. J Oral Maxillofac Surg 75: 565-575, 2017

19. Subramanian B, Krishnamurth S, Suresh Kumar P, Saravanan B, Padmanabhan M: Comparison of various approaches for exposure of infraorbital rim fractures of zygoma, J Maxillofac Oral Surg 8: 99-102, 2009.

20. Martinez AY, Bradrick JP: Y Modification of the Transconjunctival Approach for Management of Zygomaticomaxillary Complex Fractures: A Technical Note. J Oral Maxillofac Surg 70:97-101, 2012

21. Al-Moraissi EA, Seth R. Thaller b, Ellis E: Subciliary vs. transconjunctival approach for the management of orbital floor and periorbital fractures: A systematic review and meta-analysis. Journal of Cranio-Maxillo-Facial Surgery 45: 1647-1654, 2017

22. Salgarelli AC, Bellini P, Landini B, Multinu A, Consolo $\mathrm{U}$ : A comparative study of different approaches in the treatment of orbital trauma: an experience based on 274 cases. Oral Maxillofac Surg 14: 23-27, 2010.

23. Appling WD, Patrinely JR, Salzer TA: Transconjunctival approach vs subciliary skin-muscle flap approach for orbital fracture repair. Archives OtolaryngoleHead Neck Surg 119:1000-1007, 1993

24. Nunu YH, Bell A, McHugh S, Moos KF, Ayoub AF: 3D assessment of morbidity associated with lower eyelid incisions in orbital trauma. Int J Oral Maxillofac Surg 36: 680-686, 2007. 
25. Patel MP, Shapiro MD, Spinelli HM: Combined hard palate spacer graft, midface suspension, and lateral canthoplasty for lower eyelid retraction: a tripartite approach. Plast Reconstr 115: 2105-2114, 2005 discussion 2115-2117

26. Stewart JM, Carter SR: Anatomy and examination of the eyelids. Int Ophthalmol Clin 42: 1-13, 2002

27. Ridgway EB, Chen C, Colakoglu S, Gautam S, Lee BT: The incidence of lower eyelid malposition after facial fracture repair: a retrospective study and meta-analysis comparing subtarsal, subciliary, and transconjunctival incisions. Plast Reconstr Surg 124: 1578-1586, 2009

28. De Riu G, Meloni SM, Gobbi R, Soma D, Baj A, Tullio A: Subciliary versus swinging eyelid approach to the orbital floor. J Craniomaxillofac Surg 36: 439-442, 2008

29. Kelahmetoglu O, Kuzu IM, Unal M, Yagmur C, Yildiz $\mathrm{K}$, Guneren E: The comparison of complication rates of subtype subciliary approaches: The review of literature. J Exp Clin Med 33: 211-214, 2016

30. Ishida K: Evolution of the surgical approach to the orbitozygomatic fracture: from a subciliary to a transconjunctival and to a novel extended transconjunctival approach without skin incisions. J Plast Reconstr Aesthetic Surg 69: 497-505, 2016

31. Neovius E, Clarliden S, Farnebo F, Lundgren TK: Lower eyelid complications in facial fracture surgery. J Craniofac Surg 28: 391-393, 2017

32. Cole P, Kaufman Y, Hollier L: Principles of facial trauma: orbital fracture management. J Craniofac Surg 20: 101$114 ; 2009$.

33. Rohrich RJ, Janis JE, Adams Jr WP Subciliary versus subtarsal approaches to orbitozygomatic fractures. Plast Reconstr Surg 111:1708-1714, 2003.

34. Liapakis IE, Paschalis EI, Zambacos GJ, Englander M, Mandrekas AD: Redraping of the fat and eye lift for the correction of the tear trough. J. Craniomaxillofac. Surg. 42, 1497-1502, 2014

35. Bahr W, Bagambisa FB, Schlegel G, Schilli W: Comparison of transcutaneous incisions used for exposure of the infraorbital rim and orbital floor: a retrospective study. Plast Reconstr Surg 90: 585-91, 1992. 